\title{
Aportes de Vigotsky y la pedagogía crítica para la transformación del diseño curricular en el siglo XXI
}

\section{Vigotsky's contributions and critical pedagogy for the transformation of curriculum design in the twenty-first century.}

\author{
As contribuições de Vigostsky e a pedagogia crítica para a \\ transformação do desenho curricular no século XXI.
}

César Toruño Arguedas

Universidad Técnica Nacional

Alajuela, Costa Rica

ctoruno@utn.ac.cr

http://orcid.org/0000-0003-0476-7893

Recibido - Received - Recebido: 15/07/2020ＣＣＣｒregido - Revised - Revisado: 30/10/2020Ａceptado - Accepted - Aprovado: 09/11/2020

DOI: https://doi.org/10.22458/ie.v22i33.3043

URL: https://revistas.uned.ac.cr/index.php/innovaciones/article/view/3043

\begin{abstract}
Resumen: La vigencia y relevancia de los postulados de Vigotsky para la pedagogía en el siglo XXI es un hecho ante las necesidades y transformaciones sociales, políticas y económicas de sociedades que demandan una nueva respuesta educativa y, por tanto, innovaciones del diseño curricular. Ante esta realidad, el objetivo del ensayo es analizar las premisas de Vigotsky para una posible transformación del diseño curricular para impulsar su relevancia en el contexto del siglo XXI. Para tal fin, se realiza un análisis de algunas premisas académicas de Vigotsky extraídas de un análisis documental de su obra y su interacción con el currículo desde la visión de la pedagogía crítica. Se concluye que las premisas de Vigotsky sobre la superación del reduccionismo biológico, individualista y academicista del aprendizaje, la realidad sociocultural, la influencia de la estructura social en psicología del ser humano, la edad cultural de las personas estudiantes y la superación de la premisa de la población estudiantil como recipiente vacío que es llenado de contenidos; todo lo cual exige innovaciones en el diseño curricular para favorecer la pertinencia, relevancia y significancia del currículo como área de conocimiento, producto (estructura curricular) y en la realidad del aula del sistema educativo en general.
\end{abstract}

Palabras clave: Educación; currículo; diseño curricular; universidades; currículo universitario.

\begin{abstract}
The validity and relevance of Vygotsky's postulates for pedagogy in the twenty-first century is a fact given the social, political, and economic needs and social transformations that demand a new educational response, which is also true in the case of curriculum design. Faced with this reality, the present essay aims to analyze Vigotsky's premises for a potential curricular design transformation, so it is relevant in the context of the twenty-first century. Consequently, an evaluation of some of Vigotsky's
\end{abstract}


academic premises was carried out. They were examined from a critical pedagogy perspective using documentary analysis of his work and considering its interaction with the curriculum. In conclusion, Vygotsky's premise on overcoming biological, individualistic, and academic reductionism in learning, sociocultural reality, the influence of social structure in human behavior, and students' cultural age must overthrow the notion that describes students as empty containers that need to be filled with content. All of which require curricular design innovations to favor the curriculum's pertinence, relevance, and significance as a knowledge area, product (curricular structure) correspondent with classroom reality in the educational system in general.

Keywords: Education, curriculum, curriculum design, universities, higher education curriculum.

Resumo: A validade e relevância dos postulados de Vigotsky para a pedagogia no século XXI é um fato em vista das necessidades e transformações sociais, políticas e econômicas das sociedades que exigem uma nova resposta educacional e, portanto, inovações no desenho curricular. Dada esta realidade, o ensaio visa analisar as premissas de Vigotsky para uma possível transformação do desenho curricular por sua relevância no contexto do século XXI. Para este fim, é feita uma análise de algumas das premissas acadêmicas de Vigotsky extraídas de uma análise documental de seu trabalho e de sua interação com o currículo a partir da perspectiva de uma pedagogia crítica. Conclui-se que as premissas de Vigotsky sobre a superação do reducionismo biológico, individualista e acadêmico da aprendizagem, a realidade sociocultural, a influência da estrutura social na psicologia humana, a idade cultural dos estudantes e a premissa da população estudantil como um recipiente vazio que é preenchido com conteúdo; tudo isto requer inovações no desenho curricular para favorecer a pertinência, a relevância e significado do currículo como área de conhecimento, produto (estrutura curricular) e na realidade de sala de aula do sistema educacional em geral.

Palavras-chave: Educação, currículo, desenho curricular, universidades, currículo universitário. 


\section{INTRODUCCIÓN}

Los cambios culturales, políticos, tecnológicos y socioeconómicos de las últimas tres décadas han sido tan profundo como veloces y constituyen una serie de demandas de cambio, transformación y hasta revolución del diseño curricular para lograr estructuras curriculares pertinentes, relevantes y significativas para las sociedades actuales.

A partir de la realidad descrita, el presente ensayo establece la vinculación de la psicología social y la transformación del sistema educativo a partir de delimitar principios y aportes de Vigotsky y la pedagogía crítica para la transformación del diseño curricular. Por tal motivo, se establece un marco teórico curricular fundamentado en la pedagogía crítica, los insumos teóricos y prácticos de las teorías de Vigotsky a partir de una revisión de su obra y las implicaciones, en el diseño curricular, de sus aportes.

El ensayo procura establecer la vinculación práctica de los aportes de Vigotsky para facilitar la integración por parte de los diseñadores curriculares, tanto de la educación preuniversitaria como de la educación superior, con especial énfasis de la revolución curricular que implica asumir la edad cultural como referente de la organización del currículo.

Para cumplir los objetivos del presente ensayo, se desarrolla un apartado de innovaciones curriculares sustentadas en las premisas de Vigotsky que permite establecer la ruptura psicológica, epistémica y pedagógica de sus propuestas y sus respectivas implicaciones para el diseño curricular.

Lo anterior, le permitirá a la persona lectora encontrar una integración explícita entre cada aporte de Vigotsky y la repercusión curricular, específicamente los siguientes: incorporación de mecanismos para inclusión de las voces diversas en el diseño curricular, el cuestionamiento ético de la persona curriculista sobre su labor y el producto curricular en el entramado de intereses explícitos y ocultos, la necesidad de disminuir el protagonismo del fundamento psicológico en los diseños curriculares para aumentar el protagonismos de otras áreas como la sociología y la interdisciplinaridad en la formulación del diseño curricular, la reformulación de la premisa de aprendizaje por edad cronológica para dar paso a un diseño por una edad cultural y el determinante del capital, la reformulación del objetivo del diseño curricular (acumulación de contenidos o aprendizajes) y, finalmente, la adquisición de conciencia y acción sobre el diseño curricular como parte de una formación para la libertad.

\section{DESARROLLO}

El currículo es un producto cultural (Apple, 1997) construido históricamente en la dinámica de redes de poder (Kemmis, 1998) y la existencia de visiones, ideologías e intereses ocultos (Torres, 1998) que permite analizar elementos tangibles e intangibles que intervienen en los procesos de aprendizaje, desde la didáctica, la infraestructura, la oferta educativa, la estructura de la malla curricular, la organización de la estructura escolar, la distribución del poder de decisión y acción, la distribución del tiempo y las asignaturas, hasta la estructuración nacional de los procesos de contratación y selección del personal, la planificación y el presupuesto, la construcción de los programas de estudio y las políticas educativas, así como la injerencia, o no, de determinados sectores o grupos de presión.

Al concebir el currículo, resulta necesario ampliar la interpretación tradicional (asignaturas) para avanzar al análisis, desde la pedagogía crítica, de una organización del conocimiento oficial (Apple, 1996a; 1996) que plasma un modelo cultural (Gimeno, 2001), lo cual permite la visibilización de este espacio como un escenario entre la resistencia y el proyecto hegemónico (Gimeno, 1998, 1997; McLaren, 1995, Torres, 2001); es, por tanto, una forma de estructurar, sistematizar, controlar, ordenar y evaluar el sistema educativo a partir de condicionantes históricas, sociales, culturales, políticas y económicas; construida desde un 
lenguaje de esperanza (Giroux, McLaren; 1998), y de resistencia frente a la implementación de diversos proyectos hegemónicos en la era de la globalización y el neoliberalismo (Torres, 2001), economías de libre mercado, empresas transnacionales y transformación de las políticas de Estado (Apple, 1996a, 1997).

Indicado lo anterior, resulta pertinente considerar el currículo como un área de conocimiento que "investiga, analiza y sistematiza el conocimiento sobre diseño, operacionalización, evaluación y mejora de los procesos educativos dirigidos a la transmisión, reproducción, transformación y construcción de saberes, habilidades, destrezas, sensibilidades y actitudes en el marco de la educación formal" (Toruño, 2020, p. 52), caracterizado desde un posicionamiento crítico por ser "un territorio controvertido y hasta conflictivo, respecto del cual se toman decisiones, se siguen opciones y se actúa por orientaciones que no son las únicas posibles" (Gimeno, 2010, p. 29), una opción históricamente configurada "cargado, por lo tanto de valores y supuestos que es preciso descifrar" (Gimeno, 2001, p. 18), con un claro "proyecto selectivo de cultura, cultural, social, política y administrativamente condicionado, que rellena la actividad escolar, y que se hace realidad dentro de las condiciones de la escuela tal como se halla configurada" (Gimeno, 2001, p. 40)

Este currículo, se manifiesta en un currículo como proceso, producto procesado, práctica educativa, vivencia educativa, acción inesperada y el currículo reducido o formal. Por su parte, el diseño curricular debe ser considerado como:

[...] el mecanismo mediante el cual la persona curriculista logra sistematizar las cualidades de un procedimiento de formación, manifiesto en un plan de estudios o su mejora, a partir de la construcción del currículum como área del conocimiento, proceso, producto, procesado, práctica educativa, vivencia educativa, inesperado y reducido o formal; todo lo cual se sistematiza parcial o totalmente en la estructura curricular. (Toruño, 2020, p. 56)

La acción del diseño curricular descrita anteriormente debe ser comprendida desde la lectura crítica de la pedagogía crítica, a partir de procesos implícitos y explícitos para visibilizar redes de poder, intereses ocultos y proyectos hegemónicos-resistencia, así como mecanismos para la participación democrática en el diseño y el objetivo de ser instrumento para una formación dirigida a la libertad del ser humano.

Resulta necesario explicitar que la utilización de la pedagogía crítica (y por ende el currículo crítico) es el sustento pedagógico más pertinente para obtener mayores beneficios de la propuesta de procesos de aprendizaje indicados por Vigotsky. No obstante, el currículo práctico y el diseño curricular, en general, pueden obtener mayor relevancia y pertinencia con la inclusión de las premisas de Vigotsky.

Por tanto, al establecer las implicaciones de las premisas de Vigotsky para el diseño curricular, y para el currículo en general, se desarrollan principios operacionales para la innovación curricular que serán expuestas a continuación.

\section{Innovaciones curriculares sustentadas en las premisas de Vigotsky}

Al iniciar el siglo XX, Lev Vigotsky (2001) realizó una ruptura psicológica en sus textos El Problema de Desarrollo en la psicología estructural y el Método instrumental en psicología, que derivaron en una transformación epistemológica y pedagógica sustentada en la presentación de una serie de planteamientos y teorías sustentadas en el principio de que el pensamiento, el aprendizaje y las funciones "psíquicas superiores" son un producto cultural; específicamente, diversas funciones cognitivas-neurológicas son producto de la interacción del individuo con la sociedad, priorizando la construcción y el empoderamiento del lenguaje.

El mismo Vigotsky (2001, tomo II, p. 15) señala esta ruptura al indicar que el análisis atomista y funcional "dio lugar a considerar las funciones psíquicas de forma aislada y a elaborar y perfeccionar métodos de 
investigación psicológica aplicados al estudio de esos procesos aislados y separados entre sí", posiciones que serían superadas en los trabajos del autor, pero que siguen vigente en los diseños curriculares centrados obsesivamente en los aprendizajes construidos como proceso planificado (desde el currículo) e individualizado (la persona estudiante como sujeto aprende según su estructura cognitiva) y que llevan a la falsa confianza en la contextualización curricular (vivencia del aula) como laboratorio controlado de aprendizajes pretendidamente aislados de contextos del centro educativo, de los sujetos participantes y de la sociedad en general.

La superación del reduccionismo biológico, individualista y academicista del aprendizaje implica la gran transformación curricular inconclusa a casi un siglo de las propuestas de Vigotsky. La transformación se sustenta en un cuestionamiento del currículo en sí mismo para superar la visión técnica, práctica y parcialmente vigente en la visión crítica; según la cual, el currículo es una forma de planificación controlable del aprendizaje. El currículo, desde este principio de Vigotsky, es una pretensión de planificación que se sustenta, aspira y promueve la diversidad de escenarios y, por consiguiente, la variedad de aprendizajes posibles ante un mismo contenido, competencia o aspiración del diseño curricular.

Para Vigotsky "lo que determina el desarrollo ontogenético del ser humano tiene la siguiente estructura: actividad colectiva y comunicación- cultura (signos)-apropiación de la cultura (enseñanza y educación)actividad individual-desarrollo psíquico del individuo" (Chaves, 2001, p. 62); es decir, es un proceso de interacción dialéctico donde individuo-contexto interactúan y permiten la reconstrucción externa e interna del primero y las posibles alteraciones del segundo. Vigotsky (2001, tomo II, p. 59) sintetizaba esta concepción al afirmar que la "verdadera dirección del proceso de desarrollo del pensamiento del niño no va de lo individual a lo socializado, sino de los social a lo individual".

La anterior premisa implica la segunda gran transformación curricular, según la cual la persona curriculista como profesional "que plasma la interacción de fundamentos, aspiraciones pedagógicas, intereses implícitos u ocultos, realidades pedagógicas (centro educativo, comunidad y sistema) y vivencia educativa, en un documento orientador denominado estructura curricular que sistematiza la experiencia del diseño curricular" (Toruño, 2020, p. 56), requiere incorporar mecanismos para asegurar la integración de realidades socioculturales y voces diversas en el diseño curricular, asegurar la visibilidad de preguntas generadoras y cuestionadoras (el por qué y el para qué del currículo por diseñar) y, sobre todo, asegurar la flexibilidad curricular del programa de estudio para favorecer la constante interacción dialéctica entre individuo y sociedad, no como una acción inesperada, sino como una acción consciente, asumida y pensada desde la contextualización curricular.

La ruptura de Vigotsky, y los aportes de sus investigaciones, deben ser comprendidos en el marco de la influencia marxista, como afirman Barba, Cuenca y Rosa (2007, p. 5), revolucionó toda la teoría psicológica "desde las posiciones de la dialéctica materialista, hecho que le permitió aseverar que el desarrollo ocurre como resultado de la asimilación de la experiencia histórico-social y se produce gracias a la actividad y comunicación del niño con el medio social". Lo anterior, implica la tercera transformación curricular, al asumir una visión marxista, neomarxista o crítica, el diseño curricular debe manifestar en su construcción y sus productos (estructura curricular, contextualización curricular y aprendizajes) la influencia de la estructura social en psicología del ser humano, esto es posible con la integración de la pedagogía crítica y sus aportes para la visibilización de intereses, currículo oculto, proyectos hegemónicos, proyectos de resistencia, intereses de la selección y organización de contenidos, entre otros.

El análisis desde supuestos marxistas separa los postulados de Vigotsky de la interpretación psicológica tradicional (dominante en el siglo XIX e inicios del XX), sustentada en la filogenia del psiquismo; es decir, el análisis separados de lo biológico y lo social en tanto constituían entes separados; frente a esto, la propuesta de Vigotsky establece una ontogenia donde el desarrollo biológico y social (o natural y cultural) interactúan dialécticamente ya que "cuando el niño nace lo hace en un medio cultural, donde existe toda una experiencia que tendrá que asimilar" (Barba, Cuenca y Rosa, 2007, p. 6). 
La interiorización de principios marxistas queda plasmada en la siguiente afirmación:

Todas las funciones psíquicas superiores son relaciones interiorizadas de orden social, son el fundamento de la estructura social de la personalidad. Su composición, estructura genética y modo de acción, en una palabra, toda su naturaleza es social; incluso al convertirse en procesos psíquicos sigue siendo cuasi-social...Modificando la conocida tesis de Marx, podríamos decir que la naturaleza psíquica del hombre viene a ser un conjunto de relaciones sociales trasladadas al interior y convertidas en funciones de la personalidad y en formas de su estructura. (Vigotsky, 2001, tomo III, p. 151)

Establecer dicha relación, y sus implicaciones en los procesos de aprendizaje, significó una revolución en la psicología, la epistemología y el marco pedagógico en el siglo XX e implica, al iniciar el siglo XXI, la posibilidad de interpretaciones, análisis y acciones pedagógicas coherentes con posiciones de la pedagogía crítica y el progresismo político, económico y cultural. Estas premisas establecen, para el currículo, la imperiosa necesidad de concebir el diseño curricular, y el currículo en general, como un proceso pedagógico que gira en torno a seres humanos que aprenden-desaprenden en acciones subjetivas que superan la acumulación de contenidos y saberes predirigidos, todo dentro de un proceso de lucha de intereses, dominación, hegemonía, resistencia y liberación.

La relación dialéctica se traduce en una cuarta transformación al exigir un nuevo perfil del curriculista y del personal docente-administrativo de los centros educativos con una formación para el pensamiento crítico, con el objetivo de poder contextualizar el currículo y, por ende, los aprendizajes, a la influenciad el contexto comunal, regional, nacional y global de la población estudiantil. A diferencia del enfoque técnico del currículo, la premisa de Vigotsky exige un personal educativo de alto nivel crítico, capaz de generar un currículo desde las realidades de aula comprometida con las transformaciones curriculares analizadas previamente.

Se supera, por tanto, el reduccionismo individualista y academicista predominante en su época (y vigente en las estructuras curriculares occidentales al iniciar el siglo XX) y la propuesta de Piaget. En relación con las investigaciones de Piaget, el propio Vigotsky (2001, tomo 2, p. 29) reconocía que "han inaugurado una nueva era en el desarrollo de la teoría del lenguaje y el pensamiento del niño, de su lógica y su concepción de mundo". Sobre la estructura individualista-biologista de un sector de las interpretaciones de los postulados de Piaget, Vigotsky (2001, tomo II, p. 67) denunció lo siguiente:

Piaget relaciona las influencias externas y el proceso de asimilación y estudia cómo se asimilan esas influencias, es decir, son deformados por el ser vivo y las incorpora en su propia sustancia. Pero la sustancia psíquica propia del niño, la estructura y el funcionamiento característicos del pensamiento infantil, que se diferencian cualitativamente del pensamiento del adulto, están determinados, según Piaget, por el autismo, es decir por las propiedades biológicas de la naturaleza infantil. El niño no es considerado como parte del conjunto social, como sujeto de relaciones sociales que participa desde los primeros días en la vida social del conjunto a que pertenece. Lo social es considerado como algo que está fuera del niño, como una fuerza externa y extraña que le presiona y termina por desplazar sus propias formas de pensar.

Con lo anterior, Vigotsky marca la ruptura y punto de encuentro con Piaget, en el tanto reconoce la maduración cognitiva propuesta, pero advierte sobre el sesgo individualista y descontextualizado de sus posturas, en este marco es que la teoría vigostkiana ha sido denominada, generalmente como socioconstructivista; con la salvedad de que sus postulados no pueden ser interpretados como deterministas "pues trasciende al biologismo, y se atreve a afirmar que lo que diferencian al cerebro humano del cerebro 
animal es la capacidad que tiene para organizar sus funciones superiores a partir de la interacción social" (Pineda, 2011, p. 224), y establece que "la peculiaridad fundamental del desarrollo infantil radica en el entrelazamiento de los procesos de desarrollo: el cultural y el biológico" (Vigotsky, 2001, tomo III, p. 41), por tanto, el análisis y las acciones educativas vinculadas a estos postulados siempre deberán responder al contexto específico, dotándolas de especificidad temporal y espacial que impide su implementación, en forma exacta, en otro contexto o tiempo.

Además, Pineda (2011, p. 226) afirma que para Vigotsky los procesos de conocimiento "se originan en la interacción social y son exclusivamente humanos. Ellos regulan la acción en función del control voluntario y durante su organización usan formas de mediación, particularmente la semiótica", en el marco de los sistemas culturales semióticos entendidos como "la superestructura simbólica que, juntamente con la dimensión histórico-económica, da cierta forma y organización a la actividad de los individuos" (Radford, 2004 , p. 10). En este marco conceptual, Vigotsky (2001, tomo III, p. 150) concluye lo siguiente:

Podemos formular la ley genética general del desarrollo cultural del siguiente modo: toda función en el desarrollo cultural del niño aparece en escena dos veces, en dos planos; primero en el plano social y después en el plano psicológico, al principio entre los hombres como categoría interpsíquica y luego en el interior del niño como categoría intrapsíquica.

El postulado anterior permite establecer una quinta transformación del diseño curricular desde la disminución del énfasis sicologista, para dar paso a una mayor participación de la sociología, la antropología, la lingüística, la economía y la política en los estudios educativos, siendo de especial importancia los aportes de la denominada sociología educativa o sociología de la educación, en el tanto permiten establecer las características del contexto, reinterpretar las características y posibilidades de la educación como sistema formal y expanden las interpretaciones-posibilidades de los análisis de la persona, que favorezcan el cuestionamiento permanente del currículo, del proceso de aprendizaje y de la contextualización curricular.

Las implicaciones de una disminución del énfasis sicologista para pasar a una interdisciplinaridad como fundamento del diseño curricular son: a) apertura del diálogo educativo a otras profesiones y especialistas más allá de los pedagogos, b) inclusión de especialistas de diversas profesiones en la construcción de los diseños curriculares de los programas de estudio, c) cambios en la formación profesional de las personas docentes (inclusión de lo multi o transdisciplinar) y d) contratación de equipos interdisciplinarios para el acompañamiento de los procesos educativos implementados en los centros escolares.

Además, Vigotsky (2001, tomo III, p. 313) establece como principio generador de su teoría el siguiente aspecto:

El desarrollo de las funciones psíquicas superiores del niño sólo es posible por el camino de su desarrollo cultural, tanto si se trata de dominar los medios externos de la cultura tales como el lenguaje, la escritura, la aritmética, como por el perfeccionamiento interno de las propias funciones psíquicas, es decir, la formación de la atención voluntaria, la memoria lógica, el pensamiento abstracto, al formación de conceptos, el libre albedrío, etc. [...] el desarrollo cultural es la esfera más importante donde es posible compensar la insuficiencia. Allí donde el desarrollo orgánico resulta imposible, hay infinitas posibilidades para el desarrollo cultural.

Lo anterior, se complementa con la introducción del término edad cultural, la cual obliga al investigador a que "no sólo nos debemos preguntar cuál es la edad real de cada niño, cuál es su edad intelectual, sino también en qué nivel de desarrollo cultural se halla" (Vigotsky, 2001, tomo III, p. 315). Esta concepción implica una vinculación directa del análisis pedagógico con elementos sociales, económicos, culturales y sociales, permitiendo la inclusión de elementos como proyectos hegemónicos, desigualdades sociales 
y económicas, capital cultural, resistencias y otras unidades de análisis propuestas desde otras áreas disciplinares y reivindicadas desde la pedagogía crítica.

El capital cultural (pedagogía crítica) y la edad cultural (Vigotsky) son conceptos con implicaciones similares: la definición de la población estudiantil más allá de aspectos biológicos para establecer una determinación del contexto (cultura) con respecto a su estadio de desarrollo individual y cognitivo. Lo anterior se traduce en la sexta transformación del diseño curricular por cuanto ha sido un proceso constituido desde la premisa evolución lineal de aprendizajes según el cual, las personas (estudiantes) pueden adquirirconstruir conocimientos o competencias en el marco de una estructura (ciclos o grados) de acumulación de conocimientos o formación de andamiajes.

La concepción tradicional del diseño curricular omite, o al menos minimiza, el papel del contexto social, económico, cultural, político y familiar de la persona estudiante en la formación de aprendizajes y competencias. El aprendizaje de un determinado conocimiento, aprendizaje y competencia, está determinado y mediado por el contexto, por tanto, será el que brinde la pertinencia, la relevancia y la significatividad, aunado a una reinterpretación sustantiva de lo aprendido.

La edad cultural implica una revolución en el diseño curricular pues exige asumir que estudiantes de edades cronológicas iguales pueden tener diferentes estados para el aprendizaje, motivado por la influencia de la cultura en sus familias, barrios, comunidades o países. El diseño curricular requiere de lo siguiente: a) un cambio en la estructura administrativa-pedagógica de años y ciclos, b) cambios en la selección y organización de contenidos, c) una política pedagógica para la compensación de las diferencias socioculturales entre estudiantes de edades similares $y, d$ ) una flexibilidad en la estructura de evaluación para establecer requisitos de aprobación a partir de las edades culturales.

Paralelamente, Vigotsky (2001, tomo IV, p. 189) introduce un principio que será compartido por la pedagogía crítica, según el cual "no todo contenido puede incluirse en cualquier forma. El contenido no es indiferente ante la forma del pensamiento, no la colma de modo puramente externo mecánico como llena el líquido un recipiente". Este principio será reestructurado por Freire (1997) al definirlo como sistema bancario y será estudiado a profundidad por Apple $(1996,1997)$ y McLaren (1995) para establecer las implicaciones de los contenidos en los libros de textos y como parte del proyecto hegemónico.

La premisa de que la población estudiantil no es un recipiente que será rellanado con "líquido", es decir, el conocimiento; es la sétima transformación del diseño curricular del siglo XXI que puede asumirse desde las premisas de Vigotsky ya que supone el compromiso con la ruptura de una contextualización curricular dominada por prácticas tradicionales del enfoque técnico del currículo y una evaluación acumulativa, memorística y sancionatoria. Con respeto al papel de los docentes, no puede omitirse el hecho de que, en la realidad de la contextualización curricular, las personas docentes han sido reducidos a una labor técnica de reproducción de contenidos (propia del conductismo) con ligeros matices de constructivismo. No obstante, en el socioconstructivismo los docentes:

[Son responsables de...] diseñar estrategias interactivas que promuevan zonas de desarrollo próximo, para ello debe tomar en cuenta el nivel de conocimiento de las personas estudiantes, la cultura y partir de los significados que ellos poseen en relación con lo que van a aprender. (Chaves, 2011, p. 63)

Por supuesto, sin obviar la demanda desde la pedagogía crítica para convertir a las personas docentes en intelectuales transformadores (Giroux y McLaren, 1998).

Por último, se debe señalar que tanto la pedagogía crítica como los postulados de Vigotsky reconocen la importancia de la educación como proceso que facilita la construcción de conocimientos para la libertad (Freire, 2001): 
[...] sólo el concepto eleva el conocimiento de la realidad, le hace pasar del nivel de vivencia al nivel de entendimiento de la realidad, le hace pasar del nivel de vivencia al nivel de entendimiento de las leyes. Y sólo esa comprensión de la necesidad, es decir, las leyes, subyace en el libre albedrío. (Vigotsky, 2001, tomo IV, p. 200)

Todo lo anterior permite afirmar que los postulados de Vigotsky son coherentes y referentes para una propuesta de transformación del diseño curricular pues establecen cambios epistemológicos sobre la comprensión de la construcción del aprendizaje (en relación dialéctica con el contexto) y las posibilidades para la reflexión-acción del currículo como producto cultural en interacción con un contexto cultural (político, económico, social, artístico, etc.) con grupos de presión, currículo oculto e intereses tácitos de un proyecto hegemónico.

\section{SÍNTESIS Y REFLEXIONES FINALES}

El diseño curricular requiere una reingeniería en sus procesos, con el objetivo de brindar un currículo pertinente, relevante y significativo para las diversas demandas de la sociedad actual. Por este motivo, las premisas de Vigotsky y la construcción del aprendizaje pueden favorecer la innovación curricular con el valor agregado, desde un enfoque curricular crítico, al ofrecer una estructura curricular que favorezca la transformación de las realidades.

Lo expuesto en el presente ensayo permite concluir que la pedagogía crítica y el socioconstructivismo estructurado, a partir de los postulados de Vigotsky, concuerdan en la importancia de la cultura (política, economía, sociedad, etc.) en los procesos cognitivos y, esencialmente, en las posibilidades de acción y transformación educativa de los procesos de aprendizaje y formación ligados a una postura crítica.

Así mismo, los postulados de Vigotsky son una ruptura con respecto a la construcción hegemónica de la comunidad científica de su época. Dejando de lado el individualismo o la fragmentación biológico-cultura dominante en los análisis de otros autores, Vigotsky ofrece investigaciones y reflexiones que permiten ampliar y debatir los supuestos de Piaget.

La inclusión de la ontogénesis como pilar de análisis, así como la profundización de sus análisis mediante la construcción del lenguaje (semiótica), la clarificación de la relación dialéctica entre contexto y funciones superiores de la psique, la superación del reduccionismo biológico, individualista y academicista del aprendizaje, la realidad sociocultural, la influencia de la estructura social en psicología del ser humano, la edad cultural de la población estudiantil y la superación de la premisa de la persona estudiante como recipiente vacío que es llenado de contenidos, permiten establecer horizontes de análisis innovadores, pertinentes y a partir de tres ejes de reflexión-acción: contexto-educación-persona, capital cultural y edad cultural y, por último, el diseño curricular.

En el caso del diseño curricular, los principios de Vigotsky permiten establecer cambios curriculares para incorporar mecanismos de inclusión de las voces diversas en el diseño curricular, tanto en el diseño como en la contextualización curricular, el cuestionamiento ético de la persona curriculista sobre su labor y el producto curricular en el entramado de intereses explícitos y ocultos, así como la necesidad de disminuir el protagonismo del fundamento psicológico en los diseños curriculares para aumentar el protagonismos de otras áreas como la sociología y la interdisciplinaridad en la formulación del diseño curricular y la reformulación de la premisa de aprendizaje por edad cronológica. Lo anterior, para dar paso a un diseño por una edad cultural y el determinante del capital, la reformulación del objetivo del diseño curricular (acumulación de contenidos o aprendizajes) y, finalmente, la adquisición de conciencia y acción sobre el diseño curricular como parte de una formación para la libertad. 
Implícito a lo argumentado en el presente ensayo está el reconocimiento del deber de reflexión desde el progresismo educativo para asumir y operacionalizar postulados de la pedagogía crítica y de Vigotsky, así como las circunstancias socioculturales, políticas y económicas para iniciar una transformación del currículo que exige profundos esfuerzos jurídicos, académicos, administrativos y pedagógicos.

Aunado a lo anterior, es requerido profundizar las investigaciones sobre el diseño curricular desde la pedagogía crítica, implicaciones en las estructuras curriculares y contextualización curricular de los postulados de Vigotsky, así como la revolución organizacional-operacional del currículo en caso de una propuesta sustentada en la edad cultural de la persona estudiante.

\section{REFERENCIAS}

Apple, M. (1996a). Educación y poder. España: Paidós.

. (1996b). El conocimiento oficial, La educación democrática en una era conservadora. España: Paidós.

(1997). Política cultural y educación. España: Morata.

Barba, M.; Cuenca, M.; y Rosa, A. (2007). Piaget y L.S. Vigotsky en el análisis de la relación entre educación y desarrollo. En Revista Iberoamericana de Educación, 42 (7), pp. 1-12.

Chaves, A. (2001). Implicaciones Educativas de la Teoría Sociocultural de Vigotsky. En: Revista Educación. 25 (2), pp. 59-65.

Freire, P. (1977). La educación como práctica de la libertad. México: Siglo Veintiuno.

Freire, P. (2001). Pedagogía de la Indignación. España: Morata

Gimeno, J. (1997). Pedagogía por objetivos. Obsesión por la eficiencia. España: Morata.

(1998). Poderes inestables en educación. España: Morata. S.A.

(2001). El currículum: una reflexión sobre la práctica. España: Morata. S.A.

. (2010). ¿Qué significa el currículum? En Gimeno, J. (comp). Saberes e incertidumbres sobre el currículum, (pp. 21-44). Madrid: Morata

Giroux, H.; McLaren, P. (1998). Sociedad, cultura y educación. España: Niño y Dávila Editores.

Kemmis, S. (1998). El currículum: más allá de la teoría de la reproducción. España: Morata

McLaren, P. (1995). Pedagogía crítica y cultura depredadora. Políticas de oposición en la era posmoderna. España: Paidós.

Pineda, W. (2011). La teoría de la mente en la educación desde el enfoque socio-histórico de Lev Vigotsky. En Educación y Humanismo, 13 (20), pp. 220-233.

Radford, L. (2004). Semiótica cultural y cognición. Conferencia plenaria da en la XVIII Reunión Latinoamericana de Matemática Educativa. Universidad Autónoma de Chiapas. Chiapas.

Torres, J. (2001). Educación en tiempos del Neoliberalismo. España: Morata S.A.

Torres, J. (1998). El currículum oculto. España: Morata.

Toruño, C. (2020). El currículum en el contexto costarricense: propuesta de definiciones para su conceptualización. Revista Ensayos Pedagógicos, 15(1), 39-59. Recuperado de: https://doi.org/10.15359/ rep.15-1.2

Vigotsky, L. (2001). Obras Escogidas (Tomos I, II, III y IV). Madrid: Machado Libros. 